Journal of Current and Advance Medical Research

July 2019, Vol. 6, No. 2, pp. 67-68

http://www.banglajol.info/index.php/JCAMR

ISSN (Print) 2313-447X

ISSN (Online) 2413-323X

NLM Catalog ID 101673828

DOI: https://doi.org/10.3329/jcamr.v6i2.42695

EDITORIAL

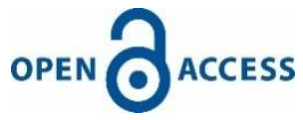

\title{
Dengue Virus and Aedes Mosquitoes: Ecological Relationship
}

\author{
AKM Shamsuzzaman
}

Professor, Department of Microbiology, Mugda Medical College, Dhaka. Former Director, Disease Control \& Line Director, Communicable Disease Control (CDC), Directorate General of Health Services, Ministry of Health and Family Welfare, Dhaka, Bangladesh; Email: zaman.tushar@gmail.com; Cell no.: +8801715566084

Human is infected by Dengue virus while female Aedes aegypti and Aedes albopictus mosquitos suck blood by biting. This is generally taken that Aedes aegypti as the primary particularly in urban and Aedes albopictus in the rural settings as secondary biological vector. Since, Aedes mosquitoes are found in tropical and sub-tropical countries, so not less than 128 countries have been facing the problems of dengue fever particularly during last 23 decades. Although, the survival period of adult Aedes mosquitoes ranges between 10 to 30 days, but usually it is considered as 21 days. Male partners undergo copulation with female once in life and then die. These female mosquitoes after copulation can store the whole population of ejaculated sperms in a safe and secured anatomical sac. From the storage the sperm comes to fertilize the eggs and thus male and female progeny are produced at a proportion of $50 \%$. A female Aedes mosquito usually lays eggs 57 times in their life span and in each event, 80 to 250 fertilized eggs are produced. Thus, a female Aedes on an average give birth to not less than 500 new female mosquitoes. A total of 2 weeks' period is needed for development and maturation of Aedes mosquito from egg to adult stage. In this way, mosquito population keep up their huge dynamic growth. Aedes mosquitoes need human blood for development, fertilization and maturation of eggs. So, female Aedes bite human beings based on a sensor maintained smell taking of human body's different components, such as lipid, aromatic hydrocarbons and sweat salts. In each occasion of biting, a single female Aedes can bite even 15 to 20 persons at a time. Even this female can impose several bites to a single individual at a single event. The more the bites, more the consumption of human blood and more the production and laying of matures eggs. Interesting to know that these female mosquitoes move from behind while make attempt to bite. During their flight, they do not make any sound. The sites of biting they choose are usually the part of upper (below elbow to hand) and lower (below knee to whole foot) extremities of human body.

The female Aedes while feeding on human blood get the adequate particles of Dengue virus. These viruses infect the mosquito's intestinal epithelial cells and undergo reproduction by the method of replication to generate huge copies of progeny positive sense RNA virus. Then those viruses are circulated to hugely infect salivary glands, from where viruses are released through saliva during biting action. Number of viruses enter into the ovary to infect ovum and continue their life cycle as transovarian passage. These mosquitoes thus act as the permanent source of Dengue virus for the rest of its life time and all the new progeny mosquitoes will have the Dengue virus. This is so important in relation to continuing generation of Dengue virus in the biological world and persisting dengue fever.

While an infected mosquito bites on human skin, the saliva is secreted containing active particles of Dengue virus. The saliva also contains an anticoagulant substance to prevent clotting and ensuring comfortable sucking of adequate amount of blood. They even continue sucking voraciously until the whole stomach is filled up. If a total number of 10 or more Dengue virus Pluck Forming Unit (PFU) per $\mathrm{mL}$ of saliva is injected in to human body even by a single bite, then there is a chance of developing Dengue fever. Thus a single mosquito bite bears the risk of Dengue virus infection. However, there are unprecedented number of mosquitoes in the environment. Hence, concerning individual living in endemic area has chances of getting many bites by single or several mosquitoes and entry of numerous virus particles. 
Aedes mosquitoes prefer to live nearest to the human habitats naturally. Mosquitos lays eggs in fresh water in different containers, such as domestics pots, households utensils, water containers, small tree harboring tubs, flower vase, empty containers, tin pot, cocoanut shell, tree holes, flag poles in the road islands, water reservoirs of under construction building, empty and broken bottles, tires of cars and so one. Wet environment is needed for at least 2 days for conversion of egg to larva. If the area becomes dried, the eggs can survive there for more than 1 year even and convert into larva while again come in contact with water. Hatched larvae can also survive in dry soil for up to three months. From larva the stage of pupa or cocoon are formed which in another 2-3 days' transform into adult mosquito.

Aedes mosquitos live inside and outside the home and bite throughout the day, especially during early morning ( 2 hours after sunrise) and late afternoon ( 2 to 3 hours before sunset). These mosquitoes also can bite at night time in well-lit area like shops and shopping malls. Since, these mosquitoes bite the back side of the likened part of the body without any sound very quickly before the person can aware of it, so man on target gets less scope to be escaped from bite. Moreover, this arthropod can bite many people at a time. Having been considering the given description, an ecological relationship between the Dengue and other Flavivirus transmission is found to be unique and this aspect needs highest emphasis while action plan is prepared to prevent and control Dengue fever outbreak.

[Journal of Current and Advance Medical Research July 2019;6(2):67-68]

[Cite this article as: Shamsuzzaman AKM. Dengue Virus and Aedes Mosquitoes: Ecological Relationship. J Curr Adv Med Res 2019;6(2):67-68] 